\section{Original} Article

\title{
Improving Survival Results after Surgical Management of Malignant Pleural Mesothelioma: An Australian Institution Experience
}

\author{
Tristan D. Yan BSc (Med), MBBS, PhD, ${ }^{1,2}$ Christopher Q. Cao, BSc (Med), MBBS, ${ }^{1,2}$ \\ Michael Boyer, MBBS, PhD, FRACP, ${ }^{3,4}$ Mo Mo Tin, MBBS, ${ }^{4,5}$ \\ Catherine Kennedy, RM, RA, , , 2 Jocelyn McLean, RN, MN, 1,2 \\ Paul G. Bannon, MBBS, PhD, ${ }^{1,2}$ and Brian C. McCaughan, MBBS ${ }^{1,2}$
}

\begin{abstract}
Background: There has been an evolving role of surgery for malignant pleural mesothelioma (MPM) over the past 25 years. The objective of this study was to investigate whether the survival results for MPM patients after surgery have improved within this time period by an analysis of a prospective cohort of 540 patients.

Methods: Five hundred and forty consecutive patients with MPM were treated by a thoracic surgical team. These patients were categorized into two groups: Group I (before September 1999, $n=270$ ) and Group II (after September 1999, $n=270$ ). The two groups were compared for clinicopathologic data and survival results. The statistical analyses of all prognostic parameters used overall survival as the endpoint.

Results: Group II had higher proportions of epithelial tumors and patients who had preoperative PET scan, extrapleural pneumonectomy (EPP), postoperative radiotherapy and pemetrexed chemotherapy. The overall survival results were significantly better in Group II compared with Group I $(p=\mathbf{0 . 0 0 4})$. Four factors were found to be independently associated with an improved survival in multivariate analysis: epithelial subtype $(p<0.001)$; surgeon's experience with $>100$ cases $(p=0.006)$, patients who underwent $\operatorname{EPP}(p=0.001)$ and those who received pemetrexed chemotherapy $(p=0.016)$. The median survival for patients selected for EPP was 20 months, as compared to 9 months for pleurodesis/decortication and pleurodesis. Conclusions: Significant improvement of overall survival results has been achieved in the more recent 270 MPM patients through accumulated experience in a specialist treatment center.
\end{abstract}

Key words: pleural mesothelioma, pleurectomy, decortication, pleurodesis and extrapleural pneumonectomy

\footnotetext{
${ }^{1}$ Department of Cardiothoracic Surgery, Royal Prince Alfred Hospital, Sydney, Australia

${ }^{2}$ The Baird Institute for Applied Heart and Lung Surgical Research, Sydney, Australia

${ }^{3}$ Department of Medical Oncology, Royal Prince Alfred Hospital, Sydney, Australia

${ }^{4}$ Sydney Cancer Center, Sydney, Australia

${ }^{5}$ Department of Radiation Oncology, Royal Prince Alfred Hospital, Sydney, Australia
}

Received: March 29, 2010; Accepted: June 22, 2010

Corresponding author: Tristan D. Yan, BSc (Med), MBBS, PhD. Department of Cardiothoracic Surgery, Royal Prince Alfred Hospital, Sydney, Australia

Email: tristan.yan@hotmail.com

Source of Financial Support: The Baird Institute for Applied Heart and Lung Surgical Research, Australia

(C)2011 The Editorial Committee of Annals of Thoracic and Cardiovascular Surgery. All rights reserved.

\section{Introduction}

The incidence of malignant pleural mesothelioma (MPM) is still increasing in most parts of the world. ${ }^{1)}$ MPM is usually associated with a median survival of 4 to 7 months for untreated cases. ${ }^{2-4)}$ In the past, the role of surgery in the management of this disease was mainly to establish the diagnosis and provide palliation of dyspnea by using pleurodesis, pleurectomy and/or decortication. ${ }^{5-7)}$ There have been no randomized trials reported, but it is likely that the survival outcomes after these palliative procedures are little different from the natural course of the disease., ${ }^{2,8)}$

Due to the locally aggressive nature of MPM, thoracic 
surgeons have focused much effort on improving the surgical techniques to provide maximal tumor clearance, and as a result, extrapleural pneumonectomy (EPP) has been utilized as the cytoreductive procedure.9) Several reports have demonstrated promising results. ${ }^{9-11)}$ However, few studies have investigated whether there is a significant improvement in the survival results in individual centers with the increasing use of a more radical treatment approach. In a rare disease like MPM, the concentration of surgical treatment in experienced thoracic centers may be a critical issue. The present study analyzes the survival outcome of 540 consecutive patients who were managed surgically in a tertiary referral center over a 25-year period, with a particular aim to determine whether there was a significant improvement in the survival outcome.

\section{Patients and Methods}

Between March 1984 to January 2008, 540 consecutive patients with MPM were treated surgically by a thoracic surgical team. Informed consent was obtained from all patients prior to surgery. The chairperson of the ethics committee has approved the current study and waived the need for patient consent for the study, as individual patients were not identified. Patient characteristics and clinical data were recorded in a prospective electronic database. Eligible patients had a tissue diagnosis of MPM confirmed by immunohistochemistry. These patients underwent extensive preoperative investigations, including a review of all prior clinical information, physical examination, serum chemistry and hematology, chest $\mathrm{x}$-ray, computed tomography (CT) of the chest and upper abdomen and pulmonary function testing. More recently, positron emission tomography (PET) was available at our institution. For the purpose of this study, the patients were categorized into two groups: Group I (before September 1999, $\mathrm{n}=270$ ) and Group II (after September 1999, $\mathrm{n}=270$ ).

There has been an evolving role of surgery for MPM at our institution over the past 25 years. The first EPP was performed in 1994, but this procedure was initially used very selectively. Prior to September 1999, 13 EPPs were performed. During this time period, in general most patients with resectable local-regional disease, absence of distant metastasis, good performance status and/or adequate pulmonary function, were considered for parietal pleurectomy, decortication and/or tumor debulking via a posterolateral thoracotomy. After September 1999, EPP was used more frequently as the en bloc resection of the disease involving the pleura, the lung, the ipsilateral hemidiaphragm and the pericardium. A total of 56 patients underwent EPP during this time period. Pleurectomy/decortication was reserved for patients with insufficient cardiopulmonary reserve, advanced age, anatomical constraints or only limited disease. In patients who were not candidates for EPP or pleurectomy/decortication, palliative pleurodesis was done either via thoracotomy or a thoracoscopic technique, and talc was instilled to cause sterile pleural inflammation and subsequent obliteration of the pleural space.

Patients with a good performance status, normal renal and liver function tests, adequate cardiac and pulmonary function assessment and the extent of disease limited to the ipsilateral hemithorax were considered for EPP. EPP was performed with en bloc resection of the lung, pleura, ipsilateral hemidiaphragm and pericardium. For a right pleuropneumonectomy, this typically involved extrapleural dissection starting posteriorly. Dissection was then continued towards the apex, allowing visualization of the mediastinal and apical structures. This was followed by mobilization of the extrapleural tissue away from the azygous vein and superior vena cava. Palpation of the diaphragm and pericardium was necessary to investigate for any involvement of tumor in these structures before they were dissected. The bronchial stump was usually reinforced circumferentially with a fat pad. Goretex reinforcement was also used in the pericardium and diaphragm. For a left pleuropneumonectomy, the procedure is similar in nature, but extra care is required to avoid injury to the intercostals arterial branches from the aorta. Details of the procedure have been described previously by Sugarbaker and colleagues. ${ }^{9)}$ Formal mediastinal lymph node dissection was performed, and all tissues were sent for histological examination. The pericardial and diaphragmatic defects were repaired with $2 \mathrm{~mm}$ Gore-Tex dual mesh.

Adjuvant radiotherapy following EPP was introduced in 2002, in an attempt to improve local-regional disease control. ${ }^{12)}$ Selection criteria for radiotherapy include good performance status, adequate residual cardio-pulmonary function and satisfactory recovery from surgery. Radiotherapy was performed within 8 to1 2 weeks of EPP. All patients underwent $\mathrm{CT}$ planning in the supine position with arms akimbo. Neither customized immobilization nor respiratory gating was used. Three dimensional conformal radiotherapy plan using a combination of photon (6 $\mathrm{MV})$ and electron beams ( $9 \mathrm{MeV}$ to $16 \mathrm{MeV})$ was generated 
for each patient. Clinical Target Volume (CTV) covers all areas of pre-operative pleural surfaces over the chest wall, mediastinum, pericardium, diaphragm including the retrocrural spaces as well as the thoracotomy and chest tube incision sites. The mediastinum was not included in the CTV in node negative patients. The planning target volume (PTV) was CTV plus $1 \mathrm{~cm}$ margin in all directions. Patients received a total dose of $45 \mathrm{~Gy}$ delivered in 25 fractions over 5 weeks to the PTV. Chemotherapy was not routinely used as an adjuvant therapy. However, in recent years some evidence suggested that pemetrexed plus cisplatin or carboplatin resulted in superior survival time. ${ }^{13-15)}$ In the present study cohort, a proportion of patients received pemetrexed combined with cisplatin or carboplatin. All patients were followed prospectively at three-monthly intervals for the first year and six-monthly thereafter until the last time of contact or death. The follow-up review included clinical examination and assessment of chest $\mathrm{CT}$ scans.

\section{Statistical analysis}

The statistical analyses of clinicopathologic parameters used overall survival as the endpoint, which was determined from the time of surgery. Survival analysis was performed by using Kaplan-Meier method and compared using the log-rank test. For multivariate analysis, a Cox-regression (Cox proportional hazards model) with forward stepwise selection of covariates and with entering and removing limits of $\mathrm{p}<0.10$ and $\mathrm{p}>0.05$ was used. Categorical variables were compared using the $\chi^{2}$ analysis or Fisher's exact test where appropriate. Mann-Whitney $U$ test was used to compare the differences between the means of continuous variables. The statistical analyses were performed by the intention-to-treat principle, using SPSS for Windows (Version 14.5; SPSS GmbH, Munich, Germany). A significant difference was assumed for $\mathrm{p}<0.05$.

\section{Results}

\section{Clinicopathologic data}

There were 455 male patients (84\%). The mean age at the time of surgery was 66 (S.D. = 11) years. Two hundred and twenty-five patients had left-sided disease, and 315 patients had right-sided disease. Two hundred and thirtyfive patients were diagnosed with epithelial subtype, and 212 patients were diagnosed with sarcomatoid or biphasic subtypes. Sixty-one patients $(11 \%)$ had a preoperative PET scan. Sixty-nine patients (13\%) underwent EPP; 269 patients (50\%) received pleurectomy/decortication and 202 patients (37\%) had palliative pleurodesis. A total of 18 patients $(3.3 \%)$ died perioperatively, including four patients who underwent EPP. Sixty-two patients (11\%) received postoperative radiotherapy for local disease control. Sixty-five patients (12\%) received pemetrexed combined with either cisplatin or carboplatin. The median follow-up period was 10 months (range 0 to 115 months), and the follow-up was complete in $96.3 \%$ of patients. At the end of the follow-up, 433 patients (80\%) were deceased. A comparison of the clinicopathologic and treatment-related data between the two groups of patients is summarized in Table 1. Group II had a significantly higher proportion of patients with epithelial subtype when compared with Group I (64\% versus 39\%, $\mathrm{p}<0.001)$. Because PET was introduced in early 2000 at our institution, no patients in Group I had a preoperative PET. When comparing Group II with Group I, there was significantly higher proportion of patients who underwent EPP ( $21 \%$ versus $5 \%, \mathrm{p}<0.001)$, who received postoperative radiotherapy $(22 \%$ versus $1 \%, \mathrm{p}<0.001)$ and who received pemetrexed combined with carboplatin or cisplatin $(23 \%$ versus $2 \%, \mathrm{p}<0.001)$. These differences reflected an increased aggressiveness of our treatment approach over the two time periods, as well as the availability of new treatment techniques. The distribution of patients were similar between the two groups, in terms of age $(p=0.639)$, gender $(p=0.813)$, the side of disease $(p=1.000)$, the presence of pleural effusion $(p=0.108)$, surgeon's experience $(\mathrm{p}=0.072)$, perioperative mortality $(\mathrm{p}=0.472)$ and the length of follow-up $(\mathrm{p}=0.418)$.

\section{Survival data}

The median survival for Group I was 9 months (range 0 to 115), with 1-, 2-, 3- and 5-year survival rates of 35\%, $16 \%, 9 \%$ and $2 \%$, respectively. The median survival for Group II was 13 months (range 0 to 72), with 1-, 2-, 3and 5-year survival rates of 50\%, 22\%, $12 \%$ and $9 \%$, respectively. Survival was significantly better in Group II than that in Group I ( $p=0.004$, Fig. 1).

Because of the significant differences in histopathologic subtype between the two groups, which might act as a confounding factor for the improved survival in Group II, a further comparison was made after stratifying patients in the two groups according to histopathologic subtype in an attempt to clarify whether the overall survival had improved for a specific subtype. As a result, significant improvement in survival was observed over the two time periods in patients with epithelial subtype 
Table 1 Comparison of clinicopathologic and treatment-related data between the initial 270 patients (Group I) and the subsequent 270 patients (Group II)

\begin{tabular}{|c|c|c|c|}
\hline Variable & $\begin{array}{c}\text { Group I } \\
\mathrm{n}(\%)\end{array}$ & $\begin{array}{c}\text { Group II } \\
\mathrm{n}(\%)\end{array}$ & $\mathrm{p}$ \\
\hline Total & 270 & 270 & - \\
\hline Age at the Time of Surgery & - & - & 0.663 \\
\hline$\leq 65$ years & $111(41)$ & $117(43)$ & - \\
\hline$>65$ years & $159(59)$ & $153(57)$ & - \\
\hline Mean \pm S.D. & $66 \pm 10$ & $67 \pm 11$ & $0.639^{*}$ \\
\hline Gender & - & - & 0.813 \\
\hline Male & $229(85)$ & $226(84)$ & - \\
\hline Female & $41(15)$ & $44(16)$ & - \\
\hline Side of Disease & - & - & 1.000 \\
\hline Left side & $112(42)$ & $113(42)$ & - \\
\hline Right side & $158(58)$ & $159(58)$ & - \\
\hline Presence of Pleural Effusion & - & - & 0.108 \\
\hline Present & $182(67)$ & $200(74)$ & - \\
\hline Absent & $88(33)$ & $70(26)$ & - \\
\hline Histopathologic Subtype & - & - & $<0.001$ \\
\hline Epithelial & $76(39)$ & $159(64)$ & - \\
\hline Sarcomatoid/biphasic & $121(61)$ & $91(36)$ & - \\
\hline Positron Emission Tomography & - & - & $<0.001$ \\
\hline Performed & $0(0)$ & $61(23)$ & - \\
\hline Not performed & $270(100)$ & $209(77)$ & - \\
\hline Surgeon's Experience & - & - & 0.072 \\
\hline Surgeons with fewer than 100 cases & $76(28)$ & $57(21)$ & - \\
\hline Surgeons with more than 100 cases & $194(72)$ & $213(79)$ & - \\
\hline Surgical Procedure & - & - & $<0.001$ \\
\hline Extrapleural pneumonectomy & $13(5)$ & $56(21)$ & - \\
\hline Pleurectomy/decortication & $172(64)$ & $97(36)$ & - \\
\hline Pleurodesis/biopsy & $85(31)$ & $117(43)$ & - \\
\hline Perioperative Mortality & - & - & 0.237 \\
\hline Yes & $11(4)$ & $7(3)$ & - \\
\hline No & $259(96)$ & $263(97)$ & - \\
\hline Postoperative Radiotherapy & - & - & $<0.001$ \\
\hline Performed & $3(1)$ & $59(22)$ & - \\
\hline Not performed & $267(99)$ & $211(78)$ & - \\
\hline Pemetrexed + Cisplatin or Carboplatin & - & - & $<0.001$ \\
\hline Performed & $4(2)$ & $61(23)$ & - \\
\hline Not performed & $266(98)$ & $209(77)$ & - \\
\hline Length of Follow-up & - & - & - \\
\hline Mean \pm S.D. & $14 \pm 17$ & $12 \pm 12$ & $0.418^{*}$ \\
\hline
\end{tabular}

* = Mann-Whitney U test

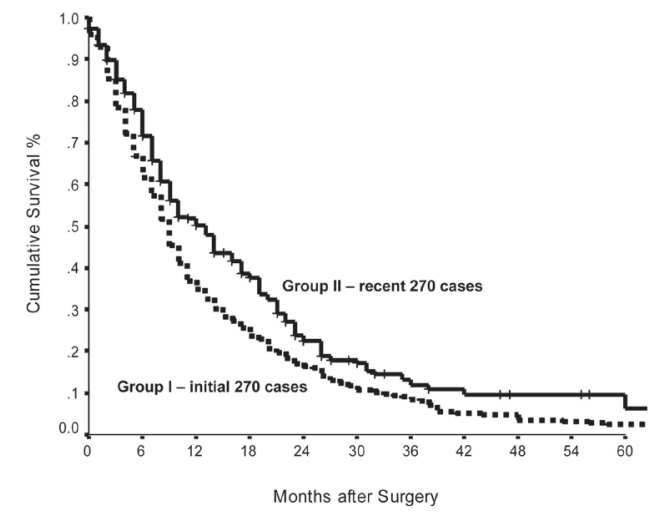

Fig. 1 Overall survival after surgical management of malignant pleural mesothelioma, stratified by study groups. Survival outcome of the more recent 270 patients (Group II) was significantly better than that of the initial 270 patients (Group I) ( $\mathrm{p}=0.004)$. $(\mathrm{p}=0.040)$, but not in those with biphasic/ sarcomatoid subtypes $(\mathrm{p}=0.176)$.

\section{Prognostic factors for survival}

The overall median survival for all 540 patients was 10 months (range 0 to 115) with 1-, 2-, 3- and 5-year survival rates of $42 \%$, $19 \%, 10 \%$ and $4 \%$, respectively. Univariate analysis identified eight prognostic factors significant for overall survival: age $\leq 65$ versus $>65$ years $(\mathrm{p}<0.001)$, epithelial versus biphasic/sarcomatoid subtypes (p <0.001) (Fig. 2), initial 270 versus subsequent 270 patients $(\mathrm{p}=0.004)$ (Fig. 1), surgeon's experience (surgeons with more than 100-case experience versus less than 100-case experience, $\mathrm{p}<0.001$ ) (Fig. 3), EPP versus pleurectomy/ decortication and pleurodesis/biopsy ( $p<0.001)$ (Fig. 4), performing preoperative PET $(\mathrm{p}<0.001)$, postoperative radiotherapy ( $\mathrm{p}<0.001)$ and pemetrexed combined with cisplatin or carboplatin $(\mathrm{p}<0.001)($ Fig. 5). Gen$\operatorname{der}(\mathrm{p}=0.058)$, left versus right side of disease $(\mathrm{p}=0.118)$ and presence versus absence of pleural effusion $(\mathrm{p}=0.143)$ did not have a significant impact on overall survival (Table 2).

These significant parameters identified in the univariate analysis were subsequently analyzed in multivariate analysis. Four factors were found to be independently associated with an improved survival: epithelial subtype (hazard ratio: 16.752; 95\% confidence interval: 1.130-1.415; $\mathrm{p}<0.001$ ); surgeon's experience with more than 100 cases (hazard ratio: 7.557; 95\% confidence interval: 1.119-1.951; $\mathrm{p}=0.006$ ); EPP (hazard ratio: 13.920; 95\% confidence interval: $1.303-2.618 ; \mathrm{p}=0.001$ ) and the use of pemetrexed containing chemotherapy (hazard ratio: 5.793; 95\% confidence interval: $0.449-$ $0.921 ; \mathrm{p}=0.016$ ).

\section{Conclusions}

The current study showed significant improvement in overall survival for patients with MPM when comparing the more recent 270 cases with the initial 270 cases. It is likely that this improvement is due to a combination of factors, such as an increased aggressive surgical approach, familiarity with the surgical procedures, introducing chemo-/radiotherapy and accumulated clinical 


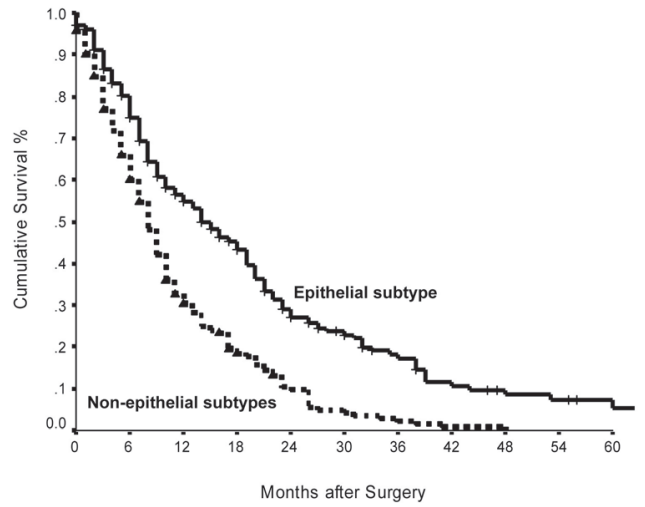

Fig. 2 Overall survival after surgical management of malignant pleural mesothelioma, stratified by histopathologic subtype. Survival outcome of epithelial subtype was significantly better than non-epithelial subtypes ( $\mathrm{p}$ $<0.001)$.

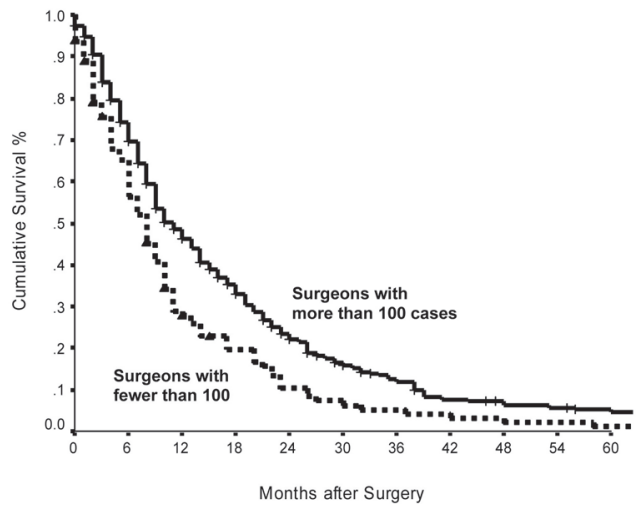

Fig. 3 Overall survival after surgical management of malignant pleural mesothelioma, stratified by surgeon's experience. Survival outcome of the patients managed by surgeons with more than 100-case experience was significantly better than those managed by surgeons with fewer than 100 -case experience $(\mathrm{p}<0.001)$.

experience. With the increased incidence of MPM and an expanded awareness of the natural history, in recent years, it has been realized that selected candidates may benefit from EPP. Several reports have demonstrated some promising survival results. ${ }^{9-11)}$ Although there have been no randomized controlled trials comparing EPP with pleurectomy/decortication, the shift into this more radical surgical approach is mainly because EPP holds more promise for achieving complete macroscopic cytoreduction, which has been considered an important determinant of prolonged survival. ${ }^{16)}$

The present study demonstrated that PET, postoperative radiotherapy and pemetrexed combination chemotherapy were significant in univariate analysis. Although the data were non-randomized, it is important to note

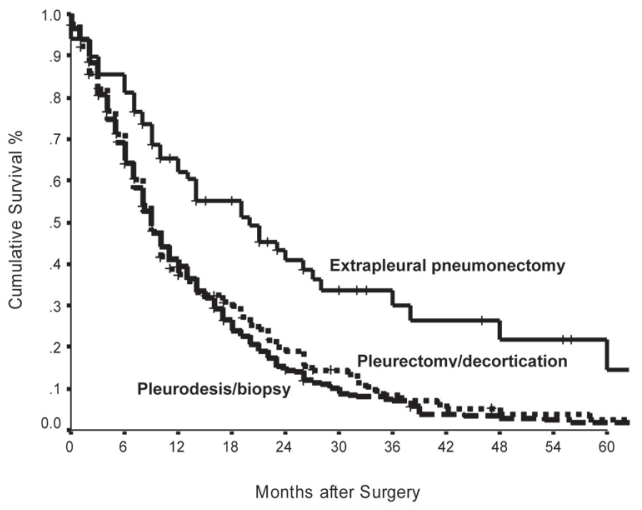

Fig. 4 Overall survival after surgical management of malignant pleural mesothelioma, stratified by operation type. Survival outcome of the patients who underwent extrapleural pneumonectomy was significantly better than those who underwent pleurectomy/decortication or pleurodesis/ biopsy $(\mathrm{p}<0.001)$.

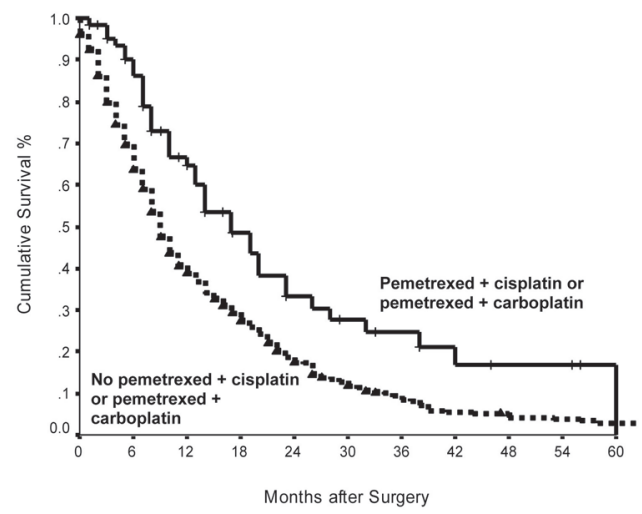

Fig. 5 Overall survival after surgical management of malignant pleural mesothelioma, stratified by adjuvant chemotherapy. Survival outcome of the patients who received adjuvant pemetrexed and cisplatin or pemetrexed and carboplatin was significantly better than those who did receive these chemotherapies $(\mathrm{p}<0.001)$.

that pemetrexed combined with cisplatin or carboplatin was also independently associated with an improved survival in the multivariate analysis. These changes in the treatment approach may have been the main reasons for the improved survival results in the recent years.

However, the comparison of the survival rates between the two groups should be interpreted with care, because there were a higher proportion of patients with epithelial histopathologic subtype in Group II. Patients with epithelial subtype have consistently been associated with an improved outcome relative to sarcomatoid or biphasic tumors. ${ }^{17-20)}$ This observation creates an impression that epithelial mesothelioma variant represents a distinct clinicopathologic entity from the other subtypes. For clarification of this confounding effect, a further comparison 
Table 2 Univariate analysis of prognostic factors affecting survival

\begin{tabular}{|c|c|c|c|}
\hline Variable & $\begin{array}{c}\text { Patient } \\
\mathrm{n}\end{array}$ & $\begin{array}{l}\text { Median Survival } \\
\text { (months) }\end{array}$ & $\mathrm{p}$ value \\
\hline Total & 540 & 10 & - \\
\hline Age at the Time of Surgery & - & - & $<0.001$ \\
\hline$\leq 65$ years & 228 & 12 & - \\
\hline$>65$ years & 312 & 9 & - \\
\hline Gender & - & - & 0.041 \\
\hline Male & 455 & 9 & - \\
\hline Female & 85 & 11 & - \\
\hline Side of Disease & - & - & 0.118 \\
\hline Left side & 225 & 10 & - \\
\hline Right side & 315 & 9 & - \\
\hline Presence of Pleural Effusion & - & - & 0.143 \\
\hline Present & 382 & 9 & - \\
\hline Absent & 158 & 11 & - \\
\hline Histopathologic Subtype & - & - & $<0.001$ \\
\hline Epithelial & 235 & 14 & - \\
\hline Sarcomatoid/biphasic & 212 & 8 & - \\
\hline Operation Periods & - & - & 0.004 \\
\hline Prior to September 1999 (Group I) & 270 & 9 & - \\
\hline After September 1999 (Group II) & 270 & 13 & - \\
\hline Positron Emission Tomography & - & - & $<0.001$ \\
\hline Performed & 61 & 19 & - \\
\hline Not performed & 479 & 9 & - \\
\hline Surgeon's Experience & - & - & $<0.001$ \\
\hline Surgeons with fewer than 100 cases & 133 & 8 & - \\
\hline Surgeons with more than 100 cases & 407 & 11 & - \\
\hline Surgical Procedure & - & - & $<0.001$ \\
\hline Extrapleural pneumonectomy & 69 & 20 & - \\
\hline Pleurectomy/decortication & 269 & 9 & - \\
\hline Pleurodesis/biopsy & 202 & 9 & - \\
\hline Postoperative Radiotherapy & - & - & $<0.001$ \\
\hline Performed & 62 & 21 & - \\
\hline Not performed & 478 & 9 & - \\
\hline Pemetrexed + Cisplatin or Carboplatin & - & - & $<0.001$ \\
\hline Performed & 65 & 17 & - \\
\hline Not performed & 475 & 9 & - \\
\hline
\end{tabular}

complete macroscopic cytoreduction is unattainable, unless the disease is minimal and localized.7) EPP, by contrast, provides more radical cytoreduction, including disease in the diaphragm and pericardium. The result of the current study demonstrated that in a large series of selected patients, EPP is independently associated with a marked improved survival. This suggests that selected patients with MPM should be considered for aggressive management. However, the procedure has been reported to be associated with moderate to high morbidity and mortality, stressing the need for careful, patient selection. ${ }^{22,23)}$ Among the 69 patients who underwent EPP, four patients died perioperatively (5.8\%). This result is perhaps acceptable for an aggressive therapy in a cohort of high-risk patients.

Multimodality therapies including cytoreductive surgery combined with adjuvant chemoradiotherapy have shown promising results. ${ }^{24,25)}$ It has to be said that currently a direct comparison of EPP versus pleurectomy/decortication with or without adjuvant chemoradiotherapy is not available, and no definitive evidence was made by stratifying patients in two groups according to the histopathologic subtype. This showed significant improvement in survival over the two time-periods only in patients with epithelial tumors. The fact that the survival outcome has not improved in the non-epithelial tumors, despite the increase in the radical treatment approach, suggests that more caution should be taken when considering these patients for EPP, so that futile aggressive treatment could be potentially avoided.

EPP requires a high level of training and expertise to optimize safety and long-term outcome for patients. For a rare disease like MPM, the concentration of services to individuals with experience is likely to increase the outcome for these patients. ${ }^{21)}$ It is important to realize that the results achieved by surgical specialists may not be replicated in routine clinical practice, and concentration of the services may improve the outcome for these patients. Whether EPP or pleurectomy/decortication is the preferred surgical approach to MPM is subject to an ongoing debate. Pleurectomy/decortication is associated with lower perioperative morbidity and mortality, but exists as to the optimal type of surgical treatment in MPM. It should also be acknowledged the difficulties of performing such trials in a rare disease like MPM. However, high quality prospective observational data collection will be extremely important and ideally, establishment of a multi-institutional registry requiring a minimum data set for all patients and recording treatment outcomes regardless of the intervention they received would provide some information on prognostically similar patients undergoing different management pathways, thereby providing some evidence for a comparison of different treatment options.

\section{Acknowledgements}

Data collection: Tristan D. Yan, Michael Boyer, Mo Mo Tin, Catherine Kennedy, Jocelyn McLean, Paul G. Bannon, Brian C. McCaughan

Statistical analysis: Tristan D. Yan, Christophe Cao

Manuscript drafting: Tristan D. Yan, Brian C. McCaughan 
Manuscript editing: Tristan D. Yan, Christopher Cao, Michael Boyer, Mo Mo Tin, Catherine Kennedy, Jocelyn McLean, Paul G. Bannon, Brian C. McCaughan

Final approval: Tristan D. Yan, Christopher Cao, Michael Boyer, Mo Mo Tin, Catherine Kennedy, Jocelyn McLean, Paul G. Bannon, Brian C. McCaughan

Financial support: Paul G. Bannon, Brian C. McCaughan

\section{Disclosure Statement}

No conflict of interest.

\section{References}

1) Robinson BW, Lake RA. Advances in malignant mesothelioma. N Engl J Med 2005; 353: 1591-603.

2) Alberts A, Falkson G, Goedhals L, Vorobiof D, Van der Merwe C. Malignant pleural mesothelioma: a disease unaffected by current therapeutic maneuvers. J Clin Oncol 1998; 6: 527-35.

3) Herndon J, Green M, Chahinian A, Corson J, Suzuki Y, et al. Factors predictive of survival among 337 patients with mesothelioma treated between 1984 and 1994 by the Cancer and Leukemia Group B. Chest 1998; 113: 723-31.

4) Rusch V. A proposed new international TNM staging system for malignant pleural mesothelioma. From the international mesothelioma interest group. Chest 1995; 108: 1122-8.

5) Brancatisano R, Joseph M, McCaughan B. Pleurectomy for mesothelioma. Med J Aust 1991; 154: 455-60.

6) Martin-Ucar AE, Edwards JG, Rengajaran A, Muller S, Waller DA. Palliative surgical debulking in malignant mesothelioma. Predictors of survival and symptom control. Eur J Cardiothorac Surg 2001; 20: 1117-21.

7) Soysal O, Karaoglanoglu N, Demircan S, Topcu S, Tastepe I, et al. Pleurectomy/decortication for palliation in malignant pleural mesothelioma: results of surgery. Eur J Cardiothorac Surg 1997; 11: 210-3.

8) Merritt N, Blewett CJ, Miller JD, Bennett WF, Young JE, et al. Survival after conservative (palliative) management of pleural malignant mesothelioma. J Surg Oncol 2001; 78: 171-4.

9) Grondin S, Sugarbaker D. Pleuropneumonectomy in the treatment of malignant pleural mesothelioma. Chest 1999; 116: 450-4.

10) de Vries WJ, Long MA. Treatment of mesothelioma in Bloemfontein, South Africa. Eur J Cardiothorac Surg 2003; 24: 434-40.

11) Allen K, Faber L, Warren W. Malignant pleural mesothelioma: extrapleural pneumonectomy and pleurectomy. Chest Surg Clin N Am 1994; 4: 113-26.

12) Yajnik S, Rosenzweig KE, Mychalczak B, Krug L, Flores R, et al. Hemithoracic radiation after extrapleural pneumonectomy for malignant pleural mesothe- lioma. Int J Radiat Oncol Biol Phys 2003; 56: 1319-26.

13) Vogelzang NJ, Rusthoven JJ, Symanowski J, Denham C, Kaukel E, et al. Phase III study of pemetrexed in combination with cisplatin versus cisplatin alone in patients with malignant pleural mesothelioma. J Clin Oncol 2003 Jul 15; 21: 2636-44.

14) Ceresoli GL, Zucali PA, Favaretto AG, Grossi F, Bidoli $\mathrm{P}$, et al. Phase II study of pemetrexed plus carboplatin in malignant pleural mesothelioma. J Clin Oncol 2006; 24: 1443-8.

15) Castagneto B, Botta M, Aitini E, Spigno F, Degiovanni $\mathrm{D}$, et al. Phase II study of pemetrexed in combination with carboplatin in patients with malignant pleural mesothelioma (MPM). Ann Oncol 2008; 19: 370-3.

16) Sugarbaker DJ. Macroscopic complete resection: the goal of primary surgery in multimodality therapy for pleural mesothelioma. J Thorac Oncol 2006; 1: 175-6.

17) Antman K, Shemin R, Ryan L, Klegar K, Osteen R, et al. Malignant mesothelioma: prognostic variables in a registry of 180 patients, the Dana-Farber Cancer Institute and Brigham and Women's Hospital experience over two decades, 1965-1985. J Clin Oncol 1988; 6: 147-53.

18) Richards WG, Zellos L, Bueno R, Jaklitsch MT, Janne PA, et al. Phase I to II study of pleurectomy/decortication and intraoperative intracavitary hyperthermic cisplatin lavage for mesothelioma. J Clin Oncol 2006; 24 : 1561-7.

19) Rusch V, Saltz L, Venkatraman E, Ginsberg R, McCormack P, et al. A phase II trial of pleurectomy/ decortication followed by intrapleural and systemic chemotherapy for malignant pleural mesothelioma. J Clin Oncol 1994; 12: 1156-63.

20) Ceresoli GL, Locati LD, Ferreri AJ, Cozzarini C, Passoni $\mathrm{P}$, et al. Therapeutic outcome according to histologic subtype in 121 patients with malignant pleural mesothelioma. Lung Cancer 2001; 34: 279-87.

21) Chowdhury MM, Dagash H, Pierro A. A systematic review of the impact of volume of surgery and specialization on patient outcome. Br J Surg 2007; 94: $145-61$.

22) de Perrot M, McRae K, Anraku M, Karkouti K, Waddell TK, et al. Risk factors for major complications after extrapleural pneumonectomy for malignant pleural mesothelioma. Ann Thorac Surg 2008; 85: 1206-10.

23) Stewart DJ, Martin-Ucar AE, Edwards JG, West K, Waller DA. Extra-pleural pneumonectomy for malignant pleural mesothelioma: the risks of induction chemotherapy, right-sided procedures and prolonged operations. Eur J Cardiothorac Surg 2005; 27: 373-8.

24) Sugarbaker D, Norberto J. Multimodality management of malignant pleural mesothelioma. Chest 1998; 113: 61-5.

25)Flores RM, Krug LM, Rosenzweig KE, Venkatraman E, Vincent A, et al. Induction chemotherapy, extrapleural pneumonectomy, and postoperative high-dose radiotherapy for locally advanced malignant pleural mesothelioma: a phase II trial. J Thorac Oncol 2006; 1: 289-95. 